November, 2006

\title{
Vacuum Birefringence in a Rotating Magnetic Field
}

\author{
Stephen L. Adler \\ Institute for Advanced Study \\ Princeton, NJ 08540
}

Send correspondence to:

Stephen L. Adler

Institute for Advanced Study

Einstein Drive, Princeton, NJ 08540

Phone 609-734-8051; FAX 609-924-8399; email adler@ias.edu 


\begin{abstract}
We calculate the vacuum polarization-induced ellipticity acquired by a linearly polarized laser beam of angular frequency $\bar{\omega}$ on traversing a region containing a transverse magnetic field rotating with a small angular velocity $\Omega$ around the beam axis. The transmitted beam contains the fundamental frequency $\bar{\omega}$ and weak sidebands of frequency $\bar{\omega} \pm 2 \Omega$, but no other sidebands. To first order in small quantities, the ellipticity acquired by the transmitted beam is independent of $\Omega$, and is the same as would be calculated in the approximation of regarding the magnetic field as fixed at its instantaneous angular orientation, using the standard vacuum birefringence formulas for a static magnetic field. Also to first order, there is no rotation of the polarization plane of the transmitted beam. Analogous statements hold when the magnetic field strength is slowly varying in time.
\end{abstract}




\section{Introduction}

There has recently been renewed interest in trying to measure the birefringence of the vacuum in a strong magnetic field predicted by the Heisenberg-Euler effective Lagrangian $[1,2,3,4]$ for quantum electrodynamics (QED). Such a measurement was a primary motivation for the PVLAS experiment [5], which however has reported a signal about $10^{4}$ times larger than expected from QED. In a recent article [6], a claim was made that just the kinematics of rotation of the magnetic field used in the PVLAS experiment, through the generation of multiple sidebands to the transmitted laser beam, could explain the PVLAS result. However, examination of ref [6] and a related paper [7] suggests several problems [8]. First, the authors have overestimated the strength of the vacuum birefringence coefficient by a factor of over 1000. Second, since on bases rotating with the magnetic field, the vacuum as modified by the magnetic field still acts on an incident electromagnetic wave as a time-independent linear medium, the presence of an infinite sequence of sidebands (as would be obtained from a nonlinear medium) is not expected; the only sidebands should be those associated with transformations between fixed laboratory bases and the rotating bases.

Nonetheless, the authors of ref [6] have called attention to an interesting, and as it turns out quite nontrivial, problem in wave propagation. This paper is devoted to formulating and solving this problem to leading order, using standard scattering theory and perturbation theory methods. Our conclusion is that to leading order in small quantities, the method used by the PVLAS group to calculate the birefringence-induced ellipticity yields the correct answer, which is independent of the rotation angular velocity $\Omega$.

To set the stage, we start from the lowest order formulas describing electromagnetic wave propagation in an external magnetic field, as deduced from the Heisenberg-Euler ef- 
fective Lagrangian. With repeated indices summed, the $\vec{d}$ and $\vec{h}$ fields are obtained from the $\vec{e}$ and $\vec{b}$ fields by

$$
\begin{aligned}
& d_{s}=\epsilon_{s t} e_{t}, \\
& h_{s}=\mu_{s t}^{-1} b_{t} .
\end{aligned}
$$

The polarization tensors $\epsilon_{s t}$ and $\mu_{s t}^{-1}$ arising from the fourth order box diagram are given by

$$
\begin{gathered}
\epsilon_{s t}=\delta_{s t}(1-2 \xi)+7 \xi \hat{B}_{s}(t) \hat{B}_{t}(t), \\
\mu_{s t}^{-1}=\delta_{s t}(1-2 \xi)-4 \xi \hat{B}_{s}(t) \hat{B}_{t}(t),
\end{gathered}
$$

with $\hat{B}_{s}(t)$ a unit vector along the magnetic field, which we take to have fixed magnitude $B$. With $B$ measured in unrationalized units, and setting $\hbar=c=1$, the parameter $\xi$ governing the strength of the QED vacuum polarization effect is given by

$$
\xi=\frac{\alpha^{2} B^{2}}{45 \pi m^{4}}
$$

where $\alpha \simeq 1 / 137.04$ is the fine structure constant and $m$ is the electron mass. Finally, in terms of $\vec{d}, \vec{h}, \vec{e}$, and $\vec{b}$ the Maxwell equations take the form

$$
\begin{aligned}
\vec{\nabla} \cdot \vec{d} & =\vec{\nabla} \cdot \vec{b}=0 \\
\vec{\nabla} \times \vec{e} & =-\frac{\partial \vec{b}}{\partial t}, \quad \vec{\nabla} \times \vec{h}=\frac{\partial \vec{d}}{\partial t}
\end{aligned}
$$

Equations (1a-d) are the fundamental physical equations governing the wave propagation problem under study. When the magnetic field orientation $\hat{B}(t)$ is time-independent, analysis of this problem shows that the vacuum becomes birefringent. Specializing to the case of a wave-vector $\vec{k}$ perpendicular to $\hat{B}$, the polarization state with $\vec{e}$ perpendicular to $\hat{B}$ propagates with index of refraction $k / \omega=1+2 \xi$, while the polarization state with $\vec{e}$ parallel to $\hat{B}$ propagates with index of refraction $k / \omega=1+\frac{7}{2} \xi$ (with $k=|\vec{k}|$ ). We will denote the difference between the larger and smaller refractive indices by $\Delta n=\frac{3}{2} \xi$. 
To finish specifying the problem, we shall assume that the linearly polarized laser beam is propagating upwards along the $z$ axis, with field strengths (expressed in terms of $(x, y, z)$ components) given by

$$
\begin{aligned}
& \vec{e}=\vec{d}=e^{i \bar{\omega}(z-t)}(\cos \theta, \sin \theta, 0), \\
& \vec{b}=\vec{h}=e^{i \bar{\omega}(z-t)}(-\sin \theta, \cos \theta, 0) .
\end{aligned}
$$

We assume that the the uniform magnetic field is confined to the region $0 \leq z \leq L$, and is oriented perpendicular to the $z$ axis, and rotates uniformly with angular velocity $\Omega$, according to

$$
\hat{B}(t)=(\cos \Omega t, \sin \Omega t, 0)
$$

from which we learn that (with the prime denoting time differentiation)

$$
\hat{B}^{\prime}(t)=\Omega(-\sin \Omega t, \cos \Omega t, 0)=\Omega \hat{z} \times \hat{B}
$$

The problem is then to calculate the upward moving transmitted wave emerging at $z=L$.

Before proceeding with details, we outline our basic strategy. The first observation is that since Eq. (2c) relates the time derivative of $\hat{B}$ to $\hat{z} \times \hat{B}$ with a time-independent coefficient, the wave propagation problem in the region $0 \leq z \leq L$ will involve differential equations with time-independent coefficients when referred to the orthonormal rotating bases $\hat{B}$ and $\hat{z} \times \hat{B}$. These differential equations can then be solved by a standard traveling wave Ansatz; this calculation of the propagation modes in the rotating magnetic field "medium" is carried out in Sec. 2. One then has to transform the incident wave to the rotating bases, and do a matching of incident, transmitted, and reflected waves at the medium boundaries $z=0$ and $z=L$. This calculation is carried out in Sec. 3. The calculation is greatly facilitated by working to leading order in the small quantities $\xi, \Omega / \bar{\omega}$, and $\Omega L$, that is, we regard both 
$\xi$ and $\Omega$ as small. Since each reflected wave is reduced in strength by $O(\xi)$, this allows us to neglect multiple reflections, such as a wave reflected back from $z=L$, and then reflected forward from $z=0$, and finally emerging at $z=L$, which makes a contribution of order $\xi^{2}$ to the transmitted wave. When multiple reflections are neglected, one can solve the wave propagation problem (to leading order) by considering independent matching problems at $z=0$ and $z=L$. Moreover, it then suffices to compute the phases of the various waves to first order in small quantities, but the corresponding transmitted wave amplitudes only to zeroth order. The reason this approximation is adequate is that the transmission coefficients at the two boundaries are independent of $L$ when multiple reflections are neglected, and so first order terms in the transmission coefficients can contribute only an $L$-independent term to the polarization parameters of the emerging wave at $z=L$. However, when $L=0$ no birefringent medium is traversed, and so the ellipticity of the emerging wave is zero; hence the first order corrections to the wave coefficients must make a vanishing contribution to the ellipticity, and similarly, to the rotation of the polarization axis. The calculation of the ellipticity and polarization axis, from our formulas for the emerging waves, is given in Sec. 4. An alternative calculation method, based on a perturbative expansion in $\xi$, is given in Sec. 5, which gives results identical to the wave-matching calculation of Sec. 3 for the rotating field case, and also applies to the case when the magnetic field strength is time dependent. A brief discussion of our results and their experimental implications is given in Sec. 6 .

\section{Electromagnetic wave eigenmodes in a rotating magnetic field}

We begin by calculating the electromagnetic wave eigenmodes in the region containing the rotating magnetic field. We write the $\vec{e}$ and $\vec{b}$ fields in terms of components along 
the rotating bases $\hat{B}$ and $\hat{z} \times \hat{B}$,

$$
\begin{aligned}
& \vec{e}=\left[E_{1}(t) \hat{B}+E_{2}(t) \hat{z} \times \hat{B}\right] e^{i k z}, \\
& \vec{b}=\left[B_{1}(t) \hat{B}+B_{2}(t) \hat{z} \times \hat{B}\right] e^{i k z} .
\end{aligned}
$$

Substituting these into Eq. (1a), and using Eq. (1b), we get the corresponding expressions for the $\vec{d}$ and $\vec{h}$ fields,

$$
\begin{aligned}
& \vec{d}=\left[(1+5 \xi) E_{1}(t) \hat{B}+(1-2 \xi) E_{2}(t) \hat{z} \times \hat{B}\right] e^{i k z} \\
& \vec{h}=\left[(1-6 \xi) B_{1}(t) \hat{B}+(1-2 \xi) B_{2}(t) \hat{z} \times \hat{B}\right] e^{i k z} .
\end{aligned}
$$

Substituting Eqs. (3a,b) into the Maxwell equations of Eq. (1d), and using Eq. (2c), we get a set of coupled equations for the coefficient functions $E_{1,2}(t)$ and $B_{1,2}(t)$,

$$
\begin{aligned}
i k E_{1}(t) & =-\Omega B_{1}(t)-B_{2}^{\prime}(t) \\
-i k E_{2}(t) & =-B_{1}^{\prime}(t)+\Omega B_{2}(t) \\
i k(1-6 \xi) B_{1}(t) & =(1+5 \xi) \Omega E_{1}(t)+(1-2 \xi) E_{2}^{\prime}(t) \\
-i k(1-2 \xi) B_{2}(t) & =(1+5 \xi) E_{1}^{\prime}(t)-(1-2 \xi) \Omega E_{2}(t) .
\end{aligned}
$$

Since this set of equations has time independent coefficients, it can be solved by making an exponential Ansatz,

$$
E_{1,2}(t)=E_{1,2} e^{-i \omega t}, \quad B_{1,2}(t)=B_{1,2} e^{-i \omega t} .
$$

Substituting Eq. (4b) into Eq. (4a), and simplifying by neglecting second order small terms proportional to $\xi^{2}$ and $\xi \Omega$, we get the coupled equations for the constant amplitudes $E_{1,2}$ and $B_{1,2}$,

$$
\begin{aligned}
k E_{1} & =\omega B_{2}+\Omega i B_{1}, \\
k i E_{2} & =-\omega i B_{1}-\Omega B_{2}, \\
k i B_{1} & =-\omega(1+4 \xi) i E_{2}+\Omega E_{1}, \\
k B_{2} & =\omega(1+7 \xi) E_{1}-\Omega i E_{2}
\end{aligned}
$$


We note at this point that we have ignored effects arising from the electric field induced by the rotating magnetic field; these are of order $\xi \Omega$ at least, and so are second order in the small parameters of the problem. Similarly, other relativistic effects associated with electrodynamics in rotating frames, on which there is a substantial literature, are not relevant for our analysis.

Since Eq. (4c) is a homogeneous set of linear equations, a solution is possible only when the determinant vanishes, which yields a quartic equation giving the dispersion relation of the wave,

$$
\left[k^{2}-\omega^{2}(1+7 \xi)\right]\left[k^{2}-\omega^{2}(1+4 \xi)\right]-2 \Omega^{2}\left(\omega^{2}+k^{2}\right)+\Omega^{4}=0
$$

This can be solved to give two solutions $k_{ \pm}$which describe upward propagating waves, and two solutions $-k_{ \pm}$which describe downward propagating waves, with $k_{ \pm}$given by

$$
\begin{aligned}
& k_{ \pm}=\omega \pm \Omega+\frac{1}{2} \omega \sigma_{ \pm} \\
& \sigma_{ \pm} \equiv \sigma_{ \pm}(\omega)=\frac{11}{2} \xi \pm\left[\left((2 \Omega / \omega)^{2}+(3 \xi / 2)^{2}\right)^{\frac{1}{2}}-2 \Omega / \omega\right]
\end{aligned}
$$

so that the corresponding refractive indices are given by

$$
k_{ \pm} / \omega=n_{ \pm}=1 \pm \frac{\Omega}{\omega}+\frac{1}{2} \sigma_{ \pm}
$$

Note that $\sigma_{ \pm}$is first order in small quantities, and is uniformly of order $\xi$ or smaller irrespective of the relative sizes of $\xi$ and $\Omega / \omega$. Also, we note that the terms of order $\xi \Omega$ that we have dropped make a contribution inside the square root of order $\xi(\Omega / \omega)^{2}$ (because the dispersion relation must be an even function of $\Omega$, as we have verified by explicit computation), and so is a higher order effect relative to the quadratic terms that we retained inside the square root. 
Solving for the ratios of the field coefficients to $E_{1}$, we get for the case of upward propagating waves with oscillatory behavior $e^{i\left(n_{ \pm} z-t\right) \omega}$

$$
\begin{aligned}
\frac{B_{2}}{E_{1}} & =1+\beta_{ \pm}, \\
\frac{i E_{2}}{E_{1}} & =\mp 1+\alpha_{ \pm}, \\
\frac{i B_{1}}{E_{1}} & = \pm 1+\gamma_{ \pm},
\end{aligned}
$$

with the quantities $\alpha_{ \pm}, \beta_{ \pm}, \gamma_{ \pm}$, which all vanish when $\xi$ vanishes, given by

$$
\begin{aligned}
\beta_{ \pm} & =\frac{7}{2} \xi \\
\alpha_{ \pm} & =\frac{\omega}{2 \Omega}(1-2 \xi)\left(7 \xi-\sigma_{ \pm}\right) \pm 2 \xi \\
& \simeq \frac{\omega}{2 \Omega}\left(7 \xi-\sigma_{ \pm}\right) \quad, \\
\gamma_{ \pm} & =-\frac{\omega}{2 \Omega}\left(2+2 \xi-n_{ \pm}\right)\left(7 \xi-\sigma_{ \pm}\right) \pm\left(2 \xi-\frac{1}{2} \sigma_{ \pm}\right) \\
& \simeq-\alpha_{ \pm},
\end{aligned}
$$

where the first lines of the expressions for $\alpha_{ \pm}$and $\gamma_{ \pm}$are accurate to first order in small quantities, and the second lines are accurate to leading zeroth order. For the case of downward propagating waves with oscillatory behavior $e^{i\left(-n_{ \pm} z-t\right) \omega}$, one simply reverses the sign of $B_{2} / E_{1}$ and $i B_{1} / E_{1}$, while keeping $i E_{2} / E_{1}$ the same as in Eqs. (6a,b). This completes the analysis of the electromagnetic wave eigenmodes in the region $0 \leq z \leq L$ containing the rotating magnetic field $B$.

\section{Wave matching to get the transmission coefficient}

Now that we know the form of propagating waves in the magnetic field region, we turn to the problem of determining the emerging waves at $z \geq L$ produced by the laser beam incident from below $z=0$. As noted in Sec. 1, when doubly reflected waves are ignored, we can solve this problem by treating sequentially the wave matching at $z=0$, followed by 
the wave matching at $z=L$. The first step is to rewrite the incident wave at $z=0$ on the rotating basis, giving

$$
\begin{aligned}
& \left.\vec{e}\right|_{z=0}=e^{-i \bar{\omega} t}[\cos (\theta-\Omega t) \hat{B}+\sin (\theta-\Omega t) \hat{z} \times \hat{B}], \\
& \left.\vec{b}\right|_{z=0}=e^{-i \bar{\omega} t}[-\sin (\theta-\Omega t) \hat{B}+\cos (\theta-\Omega t) \hat{z} \times \hat{B}],
\end{aligned}
$$

which shows that all the components of the incident wave on the rotating bases have the time dependence $e^{-i(\bar{\omega}+\Omega) t}$ or $e^{-i(\bar{\omega}-\Omega) t}$. Thus to do the match, we need a reflected wave at $z \leq 0$ with four coefficients (two for the reflected wave with frequency $\bar{\omega}+\Omega$, and two for the reflected wave with frequency $\bar{\omega}-\Omega$ ), and a transmitted wave with four coefficients (one each for the \pm eigenmodes with frequency $\omega=\bar{\omega}+\Omega$, and one each for the \pm eigenmodes with frequency $\omega=\bar{\omega}-\Omega$ ). Continuity of the $\vec{e}$ and $\vec{h}$ fields across the junction at $z=0$ gives eight matching conditions (there are two field components parallel to the boundary for each of the two fields to be matched, and two frequency components for each). Solving the wave matching equations shows, as expected, that the reflected wave amplitudes are of order $\xi$, so to get the transmitted wave amplitudes to zeroth order it suffices to ignore the reflected waves. Similarly, at $z=L$ there are also eight unknown coefficients, four for the transmitted wave (two for frequency $\bar{\omega}+\Omega$ and two for frequency $\bar{\omega}-\Omega$ ), and four for the waves of the two frequencies reflected back into the magnetic field region $z \leq L$. Again there are eight matching conditions, and solving for the reflected waves shows that they are again of order $\xi$ in magnitude, and so again the leading order coefficients of the transmitted wave can be obtained by ignoring the reflected waves.

Thus, the transmitted wave on the rotating basis, to leading order in the wave amplitudes, is simply the result of compounding transmission at $z=0$, followed by propagation from $z=0$ to $z=L$, and then followed by transmission at $z=L$. The final step is to 
convert back from the rotating bases to fixed laboratory bases. This yields three distinct emerging wave components, with frequencies $\bar{\omega}, \bar{\omega}+2 \Omega$ and $\bar{\omega}-2 \Omega$. We dispense with all of the intermediate algebra, and simply present the final result for the transmitted wave at $z=L$, which takes the form

$$
\begin{aligned}
& \left.\vec{e}\right|_{z=L}=e^{i \bar{\omega}(L-t)}(X, Y, 0), \\
& \left.\vec{b}\right|_{z=L}=e^{i \bar{\omega}(L-t)}(-Y, X, 0),
\end{aligned}
$$

with the components $X, Y$ given in terms of quantities $A, B, C, D$ by

$$
\begin{aligned}
& X=\frac{1}{2}\left(C+D+A e^{2 i \Omega t}+B e^{-2 i \Omega t}\right), \\
& Y=\frac{i}{2}\left(-C+D-A e^{2 i \Omega t}+B e^{-2 i \Omega t}\right)
\end{aligned}
$$

Introducing the definitions

$$
\begin{aligned}
\phi_{ \pm} & \equiv \frac{1}{2} \bar{\omega} \sigma_{ \pm}(\bar{\omega}), \\
a_{ \pm} & \equiv \frac{\bar{\omega}}{4 \Omega}\left[7 \xi-\sigma_{ \pm}(\bar{\omega})\right],
\end{aligned}
$$

the auxiliary quantities $A, B, C, D$ are given by the following formulas,

$$
\begin{aligned}
A & =\left(e^{i \phi_{+} L}-e^{i\left(-2 \Omega+\phi_{-}\right) L}\right) e^{-i \theta} \frac{\left(1+a_{-}\right) a_{+}}{1+a_{-}-a_{+}}, \\
B & =\left(e^{i\left(2 \Omega+\phi_{+}\right) L}-e^{i \phi_{-} L}\right) e^{i \theta} \frac{\left(1-a_{+}\right) a_{-}}{1+a_{-}-a_{+}}, \\
C & =e^{i \theta+i\left(2 \Omega+\phi_{+}\right) L} \frac{a_{+} a_{-}}{1+a_{-}-a_{+}}+e^{i \theta+i \phi_{-} L} \frac{\left(1-a_{+}\right)\left(1+a_{-}\right)}{1+a_{-}-a_{+}}, \\
D & =e^{-i \theta+i\left(-2 \Omega+\phi_{-}\right) L} \frac{a_{+} a_{-}}{1+a_{-}-a_{+}}+e^{-i \theta+i \phi_{+} L} \frac{\left(1-a_{+}\right)\left(1+a_{-}\right)}{1+a_{-}-a_{+}}
\end{aligned}
$$

These formulas are accurate to first order in small quantities in the phases, and to zeroth order in small quantities in the real amplitudes multiplying the phases. As we stressed in Sec. 1, first order corrections to the real amplitudes are independent of $L$, and so can make no contribution to physical attributes, such as the ellipticity, of the emerging wave. Expanding the exponentials through first order in small quantities, we get the following expressions for 
$A, B, C, D\left(\right.$ with $\left.\sigma_{ \pm} \equiv \sigma_{ \pm}(\bar{\omega})\right)$

$$
\begin{aligned}
& A=i \bar{\omega} L\left[\frac{2 \Omega}{\bar{\omega}}+\frac{1}{2}\left(\sigma_{+}-\sigma_{-}\right)\right] e^{-i \theta} \frac{\left(1+a_{-}\right) a_{+}}{1+a_{-}-a_{+}}, \\
& B=i \bar{\omega} L\left[\frac{2 \Omega}{\bar{\omega}}+\frac{1}{2}\left(\sigma_{+}-\sigma_{-}\right)\right] e^{i \theta} \frac{\left(1-a_{+}\right) a_{-}}{1+a_{-}-a_{+}}, \\
& C=\left[1+i \bar{\omega} L\left(\frac{2 \Omega}{\bar{\omega}}+\frac{1}{2} \sigma_{+}\right) \frac{a_{+} a_{-}}{1+a_{-}-a_{+}}+i \bar{\omega} L \frac{1}{2} \sigma_{-} \frac{\left(1-a_{+}\right)\left(1+a_{-}\right)}{1+a_{-}-a_{+}}\right] e^{i \theta}, \\
& D=\left[1+i \bar{\omega} L \frac{1}{2} \sigma_{+} \frac{\left(1-a_{+}\right)\left(1+a_{-}\right)}{1+a_{-}-a_{+}}+i \bar{\omega} L\left(-\frac{2 \Omega}{\bar{\omega}}+\frac{1}{2} \sigma_{-}\right) \frac{a_{+} a_{-}}{1+a_{-}-a_{+}}\right] e^{-i \theta} .
\end{aligned}
$$

These equations can be greatly simplified by using the following three algebraic identities obeyed by the quantities $a_{ \pm}$,

$$
\begin{aligned}
a_{+}-a_{-}+2 a_{+} a_{-} & =0 \\
\frac{\left(1-a_{+}\right)\left(1+a_{-}\right)}{1+a_{-}-a_{+}} & =1-\frac{a_{+} a_{-}}{1+a_{-}-a_{+}}, \\
1+a_{-}-a_{+} & =1+\frac{\bar{\omega}}{4 \Omega}\left(\sigma_{+}-\sigma_{-}\right) .
\end{aligned}
$$

We then get the remarkably simple formulas

$$
\begin{aligned}
& X=\cos \theta\left(1+\frac{11}{4} i \bar{\omega} L \xi\right)+\frac{3}{4} i \bar{\omega} L \xi \cos (2 \Omega t-\theta) \\
& Y=\sin \theta\left(1+\frac{11}{4} i \bar{\omega} L \xi\right)+\frac{3}{4} i \bar{\omega} L \xi \sin (2 \Omega t-\theta)
\end{aligned}
$$

which together with Eq. (8a) are our final result for the wave transmission problem. They will be used in the next section to calculate the ellipticity and major axis orientation of the emerging wave.

The vacuum with magnetic field is a specific instance of a generic weakly birefringent medium which has orthogonal polarization eigenmodes with refractive indices $n_{\|}, n_{\perp}$. Hence, with the replacements $\frac{7}{2} \xi \rightarrow n_{\|}-1,2 \xi \rightarrow n_{\perp}-1, \frac{11}{2} \xi \rightarrow n_{\|}+n_{\perp}-2$, and $\frac{3}{2} \xi \rightarrow n_{\|}-n_{\perp}$, the preceding discussion generalizes to the case in which such a generic medium is slowly rotated. 


\section{Ellipticity and polarization axis of the emerging wave}

We proceed next to calculate the polarization parameters of the emerging wave, following the exposition of Born and Wolf [9]. Let us write

$$
\begin{aligned}
& X=\cos \theta+i \Delta_{X}^{I} \simeq \cos \theta e^{i \Delta_{X}^{I} / \cos \theta} \equiv a_{1} e^{i \delta_{1}} \\
& Y=\sin \theta i \Delta_{Y}^{I} \simeq \sin \theta e^{i \Delta_{Y}^{I} / \sin \theta} \equiv a_{2} e^{i \delta_{2}}
\end{aligned}
$$

with $\Delta_{X, Y}^{I}$ first order small quantities that can be read off from Eq. (10b). Then the auxiliary angle $\alpha$ of Born and Wolf is given by

$$
\tan \alpha=\frac{a_{2}}{a_{1}}=\tan \theta
$$

which implies that $\alpha=\theta$ up to second order corrections, and so to first order there is no rotation of the polarization axis of the beam. The second auxiliary angle $\chi$ of Born and Wolf is given by $\sin 2 \chi=\sin 2 \alpha \sin \delta$; since $\delta=\delta_{2}-\delta_{1}=\Delta_{Y}^{I} / \sin \theta-\Delta_{X}^{I} / \cos \theta$ is first order small, to leading order (and allowing the ellipticity to carry a sign) we have

$$
\begin{aligned}
\Psi & =\text { Ellipticity }=\tan \chi \simeq \chi \simeq \frac{1}{2} \delta \sin 2 \alpha \simeq \frac{1}{2} \delta \sin 2 \theta \\
& =\cos \theta \Delta_{Y}^{I}-\sin \theta \Delta_{X}^{I}
\end{aligned}
$$

From the values of $\Delta_{X, Y}^{I}$ obtained from Eq. (10b), we then find

$$
\Psi=-\frac{3}{4} \xi \bar{\omega} L \sin 2(\theta-\Omega t)
$$

which using $\bar{\omega}=2 \pi / \lambda$ and $\xi=2 \Delta n / 3$ is equivalent to

$$
\Psi=-\frac{\pi L}{\lambda} \Delta n \sin 2(\theta-\Omega t)
$$

Thus, to first order in small quantities, the ellipticity is exactly what would be calculated by assuming the magnetic field to be frozen at its instantaneous position during its traversal by 
the laser beam, using the formula for ellipticity calculated from the vacuum birefringence in a static magnetic field.

\section{Perturbation method for a rotating, time-dependent magnetic field}

The simple form of the final answer of Eq. (10b) suggests there should be a simpler derivation than the wave matching procedure employed in Sec. 3. We show in this section that the same answer can be obtained by a perturbation theory approach, which while not yielding the structure of the propagation modes in the magnetic field region, has the advantage that it extends to the case when the magnetic field strength (that is, the coefficient $\xi)$ is also time dependent. Substituting the general Ansatz

$$
\begin{aligned}
& \vec{e}=E_{1}(z, t) \hat{B}+E_{2}(z, t) \hat{z} \times \hat{B}, \\
& \vec{b}=B_{1}(z, t) \hat{B}+B_{2}(z, t) \hat{z} \times \hat{B},
\end{aligned}
$$

into Eq. (1a), and using Eq. (1b) but now allowing $\xi$ to have a $t$ and $z$ dependence, we get the coupled equations

$$
\begin{aligned}
\partial_{z} E_{1}(z, t) & =-\Omega B_{1}(z, t)-\partial_{t} B_{2}(z, t) \\
-\partial_{z} E_{2}(z, t) & =-\partial_{t} B_{1}(z, t)+\Omega B_{2}(z, t) \quad, \\
(1-6 \xi) \partial_{z} B_{1}(z, t) & =(1+5 \xi) \Omega E_{1}(z, t)+(1-2 \xi) \partial_{t} E_{2}(z, t)-2 \partial_{t} \xi E_{2}(z, t)+6 \partial_{z} \xi B_{1}(z, t) \\
-(1-2 \xi) \partial_{z} B_{2}(t) & =(1+5 \xi) \partial_{t} E_{1}(z, t)-(1-2 \xi) \Omega E_{2}(z, t)+5 \partial_{t} \xi E_{1}(z, t)-2 \partial_{z} \xi B_{2}(z, t)
\end{aligned}
$$

We can solve these equations by making a perturbation expansion in powers of $\xi$, by writing $E_{1,2}=E_{1,2}^{(0)}+E_{1,2}^{(1)}+\ldots$, and similarly for $B_{1,2}$, with the fields with subscript $(0)$ the incident 
wave components on rotating bases,

$$
\begin{aligned}
& E_{1}^{(0)}=e^{i \bar{\omega}(z-t)} \cos (\theta-\Omega t), \\
& E_{2}^{(0)}=e^{i \bar{\omega}(z-t)} \sin (\theta-\Omega t), \\
& B_{1}^{(0)}=-e^{i \bar{\omega}(z-t)} \sin (\theta-\Omega t), \\
& B_{2}^{(0)}=e^{i \bar{\omega}(z-t)} \cos (\theta-\Omega t),
\end{aligned}
$$

and with the fields with superscript (1) perturbations proportional to $\xi$. The zeroth order fields are exact solutions of Eq. (12b) when $\xi$ is set to zero, but $\Omega$ is kept non-zero. Substituting the perturbation expansion into Eq. (12), differentiating $\partial_{z} E_{1,2}^{(1)}$ with respect to $z$ to get $\partial_{z}^{2} E_{1,2}^{(1)}$, and using Eq. (12b) to eliminate cross derivatives $\partial_{t} \partial_{z} B_{1,2}^{(1)}$ in terms of $E_{1,2}^{(1)}$, we get inhomogeneous wave equations for the first order perturbations,

$$
\begin{aligned}
\left(\partial_{z}^{2}-\partial_{t}^{2}\right) E_{1,2}^{(1)} & =I_{1,2} \\
I_{1} & =7 \xi \partial_{t}^{2} E_{1}^{(0)}+12 \partial_{t} \xi \partial_{t} E_{1}^{(0)}+5 \partial_{t}^{2} \xi E_{1}^{(0)}-2 \partial_{z} \xi \partial_{t} E_{1}^{(0)}-2 \partial_{z} \partial_{t} \xi E_{1}^{(0)} \\
I_{2} & =4 \xi \partial_{t}^{2} E_{2}^{(0)}+2 \partial_{t} \xi \partial_{t} E_{2}^{(0)}-2 \partial_{t}^{2} \xi E_{2}^{(0)}-6 \partial_{z} \xi \partial_{t} E_{2}^{(0)}-6 \partial_{z} \partial_{t} \xi E_{2}^{(0)}
\end{aligned}
$$

where we have dropped terms of order $\xi \Omega$ (but have kept $\Omega$ in the phases of the zeroth order solution, where it multiplies $t$, which can be arbitrarily large). Using the Green's function $G(z, t)$ for the one-dimensional wave equation with outgoing wave boundary conditions,

$$
\begin{aligned}
G(z, t) & =-\frac{1}{2} \theta(t-|z|), \\
\left(\partial_{z}^{2}-\partial_{t}^{2}\right) G(z, t) & =\delta(z) \delta(t),
\end{aligned}
$$

we can solve Eq. (13b) to give

$$
E_{1,2}^{(1)}(z, t)=\int_{0}^{L} d z^{\prime} \int_{-\infty}^{\infty} d t^{\prime} G\left(z-z^{\prime}, t-t^{\prime}\right) I_{1,2}\left(z^{\prime}, t^{\prime}\right)
$$

A similar solution can be obtained for the perturbations $B_{1,2}^{(1)}$, giving the general first order corrections to the fields, even when the magnetic field strength is $t$ and $z$ dependent. 
Let us now consider the case when the field strength is only weakly varying in time, so that $\partial_{t} \xi / \xi$ is a small parameter. Then to first order in small quantities, the terms in Eq. (13b) involving time derivatives of $\xi$ as well as explicit powers of $\Omega$ can be dropped, so that $I_{1,2}$ reduce to

$$
\begin{aligned}
& I_{1}=e^{i \bar{\omega}(z-t)} \cos (\theta-\Omega t)\left[-7 \xi \bar{\omega}^{2}+2 i \bar{\omega} \partial_{z} \xi\right], \\
& I_{2}=e^{i \bar{\omega}(z-t)} \sin (\theta-\Omega t)\left[-4 \xi \bar{\omega}^{2}+6 i \bar{\omega} \partial_{z} \xi\right] .
\end{aligned}
$$

When the magnetic field is uniform (although possibly weakly time dependent) in the interval $0 \leq z \leq L$, and zero elsewhere, one has $\partial_{z} \xi=\bar{\xi}(t)[\delta(z)-\delta(z-L)]$, with $\partial_{t} \bar{\xi}(t)$ second order small. Then substituting Eq. (15a) into Eq. (14), taking $z=L$, so that $\left|z-z^{\prime}\right|=L-z^{\prime}$ on the support of the integrand, and regarding terms of order $\Omega / \bar{\omega}$ and $\Omega L$ as small, we can evaluate the integrals over $z^{\prime}, t^{\prime}$ to get the first order fields. For the contribution from the terms $-(7,4) \xi \bar{\omega}^{2}$ in the square brackets in Eq. (15a), which have support on the interval $0 \leq z^{\prime} \leq L$, we use

$$
\begin{aligned}
& \int_{0}^{L} d z^{\prime} \int_{-\infty}^{\infty} d t^{\prime} \theta\left(t-t^{\prime}-L+z^{\prime}\right) e^{i \bar{\omega}\left(z^{\prime}-t^{\prime}\right) \pm i \Omega t^{\prime}} \\
& =\int_{0}^{L} d z^{\prime} \int_{-\infty}^{t-L+z^{\prime}} d t^{\prime} e^{i \bar{\omega}\left(z^{\prime}-t^{\prime}\right) \pm i \Omega t^{\prime}} \\
& \simeq \int_{0}^{L} d z^{\prime} \frac{i}{\bar{\omega}} e^{i \bar{\omega}(L-t) \pm i \Omega\left(t-L+z^{\prime}\right)} \\
& \simeq \frac{i L}{\bar{\omega}} e^{i \bar{\omega}(L-t) \pm i \Omega t}
\end{aligned}
$$

while for the contribution from the terms $(2,6) i \bar{\omega} \partial_{z} \xi$ in the square brackets in Eq. (15a), which have support on the field boundaries at $z^{\prime}=0, L$, we use

$$
\begin{aligned}
& \int d z^{\prime} \int d t^{\prime} \theta\left(t-t^{\prime}-L+z^{\prime}\right) \bar{\xi}\left(t^{\prime}\right)\left[\delta\left(z^{\prime}\right)-\delta\left(z^{\prime}-L\right)\right] e^{i \bar{\omega}\left(z^{\prime}-t^{\prime}\right) \pm i \Omega t^{\prime}} \\
& =\int_{-\infty}^{t} d u e^{i \bar{\omega}(L-u) \pm i \Omega u}\left[\bar{\xi}(u-L) e^{\mp i \Omega L}-\bar{\xi}(u)\right] \\
& =\mathrm{O}\left(\partial_{u} \bar{\xi}, \bar{\xi} \Omega L\right) \simeq 0
\end{aligned}
$$


Adding back the zeroth order fields, we get for the fields at $z=L$,

$$
\begin{aligned}
& \left.E_{1}\right|_{z=L}=e^{i \bar{\omega}(z-t)} \cos (\theta-\Omega t)\left(1+\frac{7}{2} i \bar{\omega} \xi L\right), \\
& \left.E_{2}\right|_{z=L}=e^{i \bar{\omega}(z-t)} \sin (\theta-\Omega t)\left(1+\frac{4}{2} i \bar{\omega} \xi L\right)
\end{aligned}
$$

which when transformed back to the fixed bases by

$$
\left.E_{1}\right|_{z=L}(\cos \Omega t, \sin \Omega t, 0)+\left.E_{2}\right|_{z=L}(-\sin \Omega t, \cos \Omega t, 0)=e^{i \bar{\omega}(z-t)}(X, Y)
$$

give

$$
\begin{aligned}
& X=\cos \theta\left(1+\frac{11}{4} i \bar{\omega} L \xi\right)+\frac{3}{4} i \bar{\omega} L \xi \cos (2 \Omega t-\theta) \\
& Y=\sin \theta\left(1+\frac{11}{4} i \bar{\omega} L \xi\right)+\frac{3}{4} i \bar{\omega} L \xi \sin (2 \Omega t-\theta)
\end{aligned}
$$

in agreement with our earlier result of Eq. (10b).

\section{Discussion}

Returning to Eq. (5b), we see that the form of the propagation eigenmodes in the magnetic field region is governed by the behavior of the square root term in $\sigma_{ \pm}$, that is, by $\left((2 \Omega / \omega)^{2}+(3 \xi / 2)^{2}\right)^{\frac{1}{2}}$, with distinctly different behaviors in the small $\Omega$ regime $2 \Omega / \omega<<$

$3 \xi / 2$, and the large $\Omega$ regime $2 \Omega / \omega>>3 \xi / 2$. In the former, the refractive indices are $n_{+} \simeq 1+(7 / 2) \xi$ and $n_{-} \simeq 1+2 \xi$, as in the case of a non-rotating magnetic field. In the latter, the refractive indices are $n_{ \pm} \simeq 1+(11 / 4) \xi \pm \Omega / \omega$. This change in character of the eigenmodes does not show up in the transmitted wave polarization parameters, however, because all dependence on the square root cancels between the various terms in Eq. (11a). The simple results that we get for the polarization parameters are what one would immediately get by assuming that the dependence on $\xi$ and $\Omega$ should be analytic around the origin in these parameters, since then the fact that effects of the magnetic field region vanish at $\xi=0$ 
implies that there can be no first order term proportional to $\Omega$; the rotation dependence must first enter at second order through terms proportional to $\xi \Omega$. Similarly, dependences on a small time rate of change of the magnitude of the magnetic field, which are relevant for experiments in which a non-rotating but ramping magnetic field is used, must also appear only in second order terms in small parameters.

We note in conclusion that putting in the numbers characterizing the PVLAS experiment, even though their magnetic field rotates only a fraction of a revolution per second, their parameters yield $\Omega L=0.7 \times 10^{-8}, 2 \Omega / \bar{\omega} \simeq 2 \times 10^{-15}$, and $3 \xi / 2 \simeq 10^{-22}$. So although all of these are small, PVLAS is in fact operating in the large $\Omega$ regime in terms of the behavior of the square root and the propagation eigenmodes in the rotating field region. Nonetheless, as we have shown, the formulas they have used for the ellipticity and polarization axis orientation of the emerging wave are correct.

\section{Acknowledgments}

This work was supported in part by the Department of Energy under Grant \#DEFG02-90ER40542. I wish to thank Raúl Rabadán for bringing the paper of ref [6] to my attention, and participants in the "Axions at the Institute for Advanced Study" workshop for stimulating discussions. After the initial draft of this paper was posted to the arXiv, I benefited from informative email correspondence with Carlo Rizzo and Holger Gies, and from helpful referee comments.

Added note: After this work was completed, we learned of related work by Biswas and Melnikov [10], whose results agree with ours. 


\section{References}

[1] Heisenberg W and Euler H (1936) Z. Physik 98714

[2] Schwinger J (1951) Phys. Rev. 82664

[3] Toll J (1952) unpublished Princeton dissertation

[4] Adler S L (1971) Ann. Phys. 67599

[5] Zavattini E et al. (2006) Phys. Rev. Lett. 96 110406; Bakalov D et al. (1994) Nucl. Phys. B Proc. Suppl. 35 180; Bakalov D et al. (1998) Quantum Semiclass. Opt. 10 239; Bakalov D et al. (1998) Hyperfine Interact. 114103.

[6] Tito Mendonça J, Dias de Deus J and Castelo Ferreira P (2006) Phys. Rev. Lett. 97 100403

[7] Tito Mendonça J, Dias de Deus J and Castelo Ferreira P (2006) arXiv:hep-ph/0609311

[8] Problems with the calculation of ref [6] were first pointed out by Holger Gies (unpublished memorandum, July, 2006), and problems with ref [6] and ref [7] were noted independently 
by the author (unpublished memorandum, October, 2006)

[9] Born M and Wolf E (1964) Principles of Optics (New York:Macmillan); for a concise exposition with application to PVLAS, see

Adler, S L (2006) http://www.sns.ias.edu/ adler/talks.html

[10] Biswas S and Melnikov K (2006) arXiv: hep-ph/0611345 